\title{
Adenoviral Transfer of mda-7 Leads to BAX Up-regulation and Apoptosis in Mesothelioma Cells, and is Abrogated by Over-expression of BCL-XL
}

\author{
Xiaobo X. Cao, ${ }^{1}$ Imran Mohuiddin, ${ }^{1}$ Sunil Chada, ${ }^{3}$ Abner M. Mhashilkar, ${ }^{3}$ Mustafa K. Ozvaran, ${ }^{1}$ \\ David J. McConkey ${ }^{2}$ Steven D. Miller, ${ }^{1}$ Jonathon C. Daniel, ${ }^{1}$ and W. Roy Smythe ${ }^{1}$ \\ ${ }^{1}$ Department of Thoracic and Cardiovascular Surgery, Section of Thoracic Molecular Oncology \\ ${ }^{2}$ Department of Cancer Biology, The University of Texas M. D. Anderson Cancer Center \\ ${ }^{3}$ Introgen Therapeutics, Inc., Houston, TX, USA \\ Accepted October 22, 2002
}

\begin{abstract}
Background: Malignant pleural mesothelioma (MPM) is unresponsive to conventional therapies. Forced expression of the novel tumor suppressor mda-7 gene in other cell types has resulted in decreased growth and apoptosis. We evaluated cell growth, apoptosis and tumor suppressor characteristics following forced expression of this gene in mesothelioma cell lines.

Methods: MDA-7 expression in human MPM cells at baseline, following pharmacologic differentiation and viral mda-7 transduction (Ad-mda7) were evaluated with Western blot. Cell viability was evaluated with a colorimetric (XTT) assay, and apoptosis with subGl FACS and Hoescht. Caspase-3 expression was evaluated by functional assay. These parameters were also evaluated in a stable bcl-xl hyper-expressing MPM
\end{abstract}

cell line. Bax mRNA levels were evaluated with realtime PCR.

Results: No baseline or differentiated MPM MDA7 expression was found, but was noted following Ad-mda7 exposure. More than $50 \%$ of MPM cells were killed at 5 days following Ad-mda7 exposure $(p<0.001)$. Apoptosis was accompanied by caspase- 3 cleavage and increased BAX expression at both the protein (translational) and mRNA (transcriptional) level. These findings were reduced in a bcl-xl hyper-expressing cell line $(\mathrm{P}<0.01)$.

Conclusions: Although mda-7 does not appear to be a MPM suppressor gene, adenoviral-mediated expression in cell lines induces apoptotic cellular death related to BAX upregulation and caspase cleavage. This is supported by abrogation of effect in a bcl-xl hyper-expressing cell line.

\section{Introduction}

Malignant pleural mesothelioma (MPM) is a solid neoplasm that originates from the parietal and visceral pleural surfaces. This tumor is extremely unresponsive to conventional therapies, with a systemic chemotherapy response rate of less than $20 \%$, and an anticipated survival of no more than two years from the time of diagnosis (1-3). The unresponsiveness of this tumor to conventional treatment has engendered a recent interest in novel approaches such as viral vector mediated gene therapy. Transfer of the pl6 gene, the herpes simplex thymidine kinase gene (used in a suicide gene paradigm with ganciclovir as a prodrug), and various immunoregulatory protein genes to MPM cells and tumors have been attempted (4-7). At least one of these gene therapy approaches has been introduced clinically in a completed phase I trial (8). Although cytogenetic abnormalities are not unusual in MPM, the use of gene replacement

Address correspondence and reprint requests to: W. Roy Smythe, Department of Thoracic and Cardiovascular Surgery,

The University of Texas M.D. Anderson Cancer Center,

1515 Holcombe Boulevard, Box 109, Houston, Texas 77030,

Phone: 713-792-6933, email: rsmythe@mdanderson.org. therapies has not been embraced because mesothelioma tumors and cell lines do not display mutations in "classical" growth control genes such as p53 and ras (9-11).

The artificial induction of apoptosis via gene therapy techniques is another potential area of investigation. Unfortunately, an understanding of the mechanisms by which this tumor maintains apoptotic homeostasis is lacking, and along with it, potential targets in the various apoptotic pathways. Investigators have determined that the anti-apoptotic bcl-2 gene is relatively infrequently expressed, but expression of the bcl-xl gene is rarely, if ever, absent $(12,13)$. The expression of the pro-apoptotic genes bax and bak seem also to be somewhat uniform, but little is known about the expression of TRAIL and related proteins, various caspases and other mediators of apoptosis.

Exclusive of members of known apoptotic families, there are agents which seem to initiate apoptosis by unrelated mechanisms, even though the ultimate death effector pathways may be similar, and the mda- 7 gene product falls into this category. The mda-7 gene was identified by subtraction hybridization from the HO-1 melanoma cell line by 
Jiang, et al. in 1995. The gene codes for a novel evolutionarily conserved $23.8 \mathrm{kD}$ protein consisting of 206 amino acids (14). mda-7 mRNA was not expressed in melanoma cells, but mda-7 mRNA was increased when melanoma cells were differentiated with beta-interferon and mezerin. The mda-7 gene was also expressed in primary melanocyte cultures. Later studies have suggested that the gene is constitutively active, and that post-transcriptional processes may be responsible for lack of mRNA expression in this particular tumor type (15). MDA-7 protein expression is found in primary melanocyte cultures, and in normal human skin melanocytes. However, MDA-7 protein expression is decreased in advanced melanomas, and is virtually absent in metastatic disease (16). Forced expression of the mda-7 gene product in melanoma cells resulted in decreased growth potential, and this has been repeated for a number of other tumor types, utilizing both adenoviral as well as plasmid gene transfer (17-21). The data thus far suggest that the mda-7 gene acts as a tumor suppressor, but a unifying mechanism of action has not been described.

In this study we demonstrate that adenoviral transfer of the mda-7 transgene leads to apoptotic cellular death in human MPM cells - a tumor type usually quite resistant to apoptotic stimuli. The mechanism by which this process occurs is not certain, but appears to be p53 independent, associated with increased BAX protein and mRNA expression and subsequent cytoplasmic caspase activation, and is abrogated with over-expression of the bcl-xl gene product.

\section{Materials and Methods}

Cell Lines, Conditions, Growth and Differentiation with Sodium Butyrate

The human MPM cell line I-45 (sarcomatous subtype, p53 wild type) was originally provided by Dr. Joe Testa of the Fox Chase Cancer Center in Philadelphia, PA. The REN (inflammatory epithelial subtype, p53 mutant) cell line was developed by the primary author (W.R.S.) and has been described elsewhere (5). Cell lines were maintained in RPMI 1640 media with $10 \%$ fetal bovine serum (Life Technologies, Inc., Gaithersburg, MD) and antibiotics.

For experiments involving differentiation, cells were exposed to sodium butyrate. Each cell line was seeded unto a six well plate with $5.0 \times 10^{5}$ cells per well. Cells were incubated for 24 hours to allow adherence and then $3 \mathrm{mM}$ of sodium butyrate (Sigma Chemical Co., St. Louis, MO) was added to the study wells and re-incubated for 48 hours. Media was then aspirated from the wells and the cells were washed with PBS and lysates collected as described below.

\section{Western Blot Analysis}

Cell lysates were prepared 72 hours following Admda7 exposure by lysing plated cell monolayers with SDS-PAGE sample buffer. The protein concentration was then determined by BCA protein assay (Pierce, Rockford, IL). SDS-Polyacrylamide ( $10 \%$ or $12 \%)$ gels were loaded with 50 ug of cell lysate. After electrophoresis, the proteins were transferred to high bond-ECL membranes. The membranes were then incubated with the primary and secondary antibodies, and developed according to the Amersham ECL protocol. Actin, caspase, BCL-XL, BAK, and BAX antibodies were obtained from Santa Cruz Biotechnology, Inc. (Santa Cruz, CA). BCL-2 antibody was obtained from Dako Corporation (Carpenteria, CA). MDA-7 protein was detected using an affinity-purified polyclonal ant-MDA-7 sera raised against bacterially-expressed MDA-7 (21). Antibody dilutions were as follows: BAX 1:500, BCL2 1:2000, BCL-XL 1:500 and Actin 1:3000. Incubation times were 3 hours at room temperature for all primary antibodies. Secondary antibodies (anti-mouse IgG and anti-rabbit IgG peroxidase linked) were incubated at room temperature for 1 hour at a dilution of $1: 1000$.

\section{Real-Time Quantitative PCR Analysis}

I-45 cells were transfected with Ad-mda7 or Ad-Luc (marker vector capable of transferring the firefly luciferase gene) at 3000 virus particle per cell. After 72 hours virus infection, total RNA was extracted with RNAqueous small scale RNA isolation kit (Ambion Inc, Austin, TX). Reverse transcription was carried on with Taqman Reverse Transcription Kit (Applied Biosystems, Foster City, CA). For quantitative real-time RT-PCR, human total RNA was used as a relative standard and human GAPDH gene was used as an internal control for relative mRNA amount. Real-time PCR was performed in the ABI Prism 7700 Sequence Detection System (Applied Biosystems) according to the protocol of the manufacturer. Typical amplification mixes $(25 \mathrm{ml})$ contained the sample DNA (or cDNA), 10X TaqMan Buffer $(2.5 \mathrm{ml}), 200 \mathrm{mM}$ dATP, dCTP, dGTP, and $400 \mathrm{mM}$ dUTP, $5 \mathrm{mM} \mathrm{MgCl}$, 0.65 units of AmpliTaq Gold, 0.25 units of AmpErase uracil N-glycosylase (UNG), $200 \mathrm{nM}$ each primer and $100 \mathrm{nM}$ probe. The thermal cycling conditions consist of 1 cycle at $2 \mathrm{~min}$ for $50^{\circ} \mathrm{C}$ and $10 \mathrm{~min}$ for $95^{\circ} \mathrm{C}$, and 50 cycles of $95^{\circ} \mathrm{C}$ for $15 \mathrm{~s}$ and $60^{\circ} \mathrm{C}$ for $1 \mathrm{~min}$. All reactions were performed in quadriplicates. After the reaction, the build-in software in the 7700 system analyses all the data and generates the standard curve, the $\mathrm{Ct}$ value of each testing sample and their corresponding starting quantity based on the standard curve.

Bax forward primer: AGTAACATGGAGCTGCAGAGGAT Bax reverse primer: GCTGCCACTCGGAAAAAGAC Bax TaqMan probe: TTGCCGCCGTGGACACAGACTC

Cell Viability Assay

The XTT kit was used to determine cell proliferation. Briefly, cells were plated in 96 well plate at a 
concentration of 2000 cells per well. Cells were then transduced with Ad-mda7 adenovirus or control Ad-lacZ (3000 vp/cell). After incubating for 96 hours, the XTT reaction mixture which contains XTT labeling reagent and the electron-coupling reagent in a 20:1 ratio was added to each well. Finally, wells were evaluated using the colorimetric plate reader at a wavelength of $450 \mathrm{~nm}$. Each sample was assayed in triplicate fashion. Data was then analyzed using Microsoft EXCEL.

\section{Recombinant Adenovirus and In Vitro Gene Transfer}

The Ad-mda7 vector was constructed and characterized as described previously (19). An adenoviral vector capable of transferring the marker gene E. coli beta-glucuronidase was utilized as a control. All viral preparations were found to be free of ElA adenovirus region and endotoxin. The virus particle (vp) titer was determined by the absorbency of the dissociated virus at A260 nm. As determined in preliminary experiments, I-45 and REN were transfected with Ad-lacZ and Ad-mda7 at 3000 viral particles/cell per to ensure $90 \%$ transfection efficiency.

\section{DNA Content Analysis and Hoescht Staining for Apoptosis}

Apoptotic cell death was evaluated by changes in cell morphology and flow cytometry. Fluorescenceactivated cell sorter analysis was performed as follows: following 96 hours of exposure to a one time dose of 3000 viral particles/cell of either Ad-mda7 or Ad-lacZ, cells were trypsinized, collected by centrifugation, resuspended in PBS and fixed in $70 \%$ ethanol at $4^{\circ} \mathrm{C}$ overnight. After centrifugation, the cells were washed in PBS and resuspended in PI staining solution (Boehringer Mannheim Co, Indianapolis, IN). Specimens were incubated in the dark for $30 \mathrm{~min}$ at $37^{\circ} \mathrm{C}$, and analyzed with the use of an EPICS Profile II flow cytometer (Coulter corp., Hialeah, FL). An analysis region was set based on the negative controls, and the percentage of sub-Gl cells was calculated from this region.

For Hoescht staining, cells were plated in chamber slides using a concentration of 5000 cells in $500 \mu \mathrm{L}$ of media. After 24 hours, the cells were exposed to 3000 viral particles/cell of Ad-mda7 or Ad-lacZ. After 5 days, cells were washed with PBS and then fixed with acetone/acetic acid (3:1). Following fixation, Hoechst nuclear staining solution $(0.1 \mu \mathrm{g} / \mathrm{mL})$ was added. Nuclei were than examined using the fluorescent microscope (Nikon Diaphot: Nikon Inc, Melville, NY). Apoptosis was characterized as cells with large segmented nuclei.

\section{Caspase-3 Functional Assay}

Three million cells were plated on a $10-\mathrm{cm}$ dish and incubated for 24 hours. After incubation, the cells were then treated with Ad-mda7 or Ad-lacZ for 72 hours. Next, cells were washed with PBS, and lysed using $10 \mathrm{~mL}$ of Cell Lysis Buffer (Pharmingen, San Diego, CA), and then transferred in $100 \mu \mathrm{L}$ aliquots to $1.5 \mathrm{~mL}$ Eppendorf tubes. For each reaction, $10 \mu \mathrm{L}$ of the substrate Ac-DEVD-AMC (Pharmingen, San Diego, CA) and $1 \mathrm{~mL}$ of HEPES buffer (Pharmingen, San Diego, CA) were added. The reaction mixtures were incubated at $37^{\circ} \mathrm{C}$ for 1 hour. The reaction mixture was then transferred to a 96 well plate. Following this, the fluorescent AMC liberated from the reaction was measured using a spectrofluorometer (Dynatech Labs, Chantily, VA) at an excitation wavelength of $380 \mathrm{~nm}$ and an emission wavelength of $440 \mathrm{~nm}$. An inhibitory reaction was set up using the 4 sets of cell lysates as described previously. Ac-DEVD-AMC $(10 \mu \mathrm{L})$ as combined with $10 \mu \mathrm{L}$ of the inhibitory Ac-DEVD-CHO (Pharmingen, San Diego, CA) and then added to ImL of HEPES buffer making a new reaction buffer. This new reaction mixture was then combined with $100 \mu \mathrm{L}$ of each cell lysate in a $1.5 \mathrm{~mL}$ Eppendorf tube. Fluorescent activity was measured as previously described.

\section{Plasmid and DNA Transfection}

The plasmid pcDNA3.1/BCL-XL was constructed by inserting human bcl-xl cDNA into an EcoRl site of the pcDNA 3.1 vector (Introgen, Houston, TX). This bcl-xl plasmid was kindly provided by Dr. David McConkey of The University of Texas M. D. Anderson Cancer Center. The pd2EGFP-NI vector plasmid (for generation of stable green fluorescent proteinexpressing mesothelioma clones to serve as controls) was purchased from Clontech (Clontech Laboratories, Inc; Palo Alto, CA). The I-45/GFP(I-45gfp) and I-45/BCL-XL(I-45xl) were generated by transfecting parental I-45 cells with pd2EGFP-N1 and pcDNA3.1/BCL-XL respectively using FUGENE-6 transfection reagent (Roche Molecular Biochemicals, Indianapolis, IN). Cells were then selected with $400 \mu \mathrm{g} / \mathrm{ml} \mathrm{G} 418$. Single cell clones were isolated and further cultured in G418 medium. Stable overexpressing clones were confirmed for level of BCLXL protein expression by Western blot analysis and densitometry.

\section{Results \\ Human Mesothelioma Cells do not Express Endogenous MDA-7 Protein, and MDA-7 is not Induced by Differentiation}

Protein lysates for the REN and I-45 cell lines were collected, and Western blot analysis was performed to evaluate endogenous MDA-7 protein expression. No expression of the MDA-7 was noted in untreated cells (Fig. 1). We evaluated expression of MDA-7 protein following exposure of MPM cells to the differentiating agent sodium butyrate. This histone deacetylase inhibitor has been shown by our group to differentiate MPM cells in vitro at a dose of $3 \mathrm{mM}$, with increased alkaline phosphatase expression and development of a more differentiated morphology (22). Differentiation of MPM cells did not activate MDA-7 protein expression (Fig. 1). 


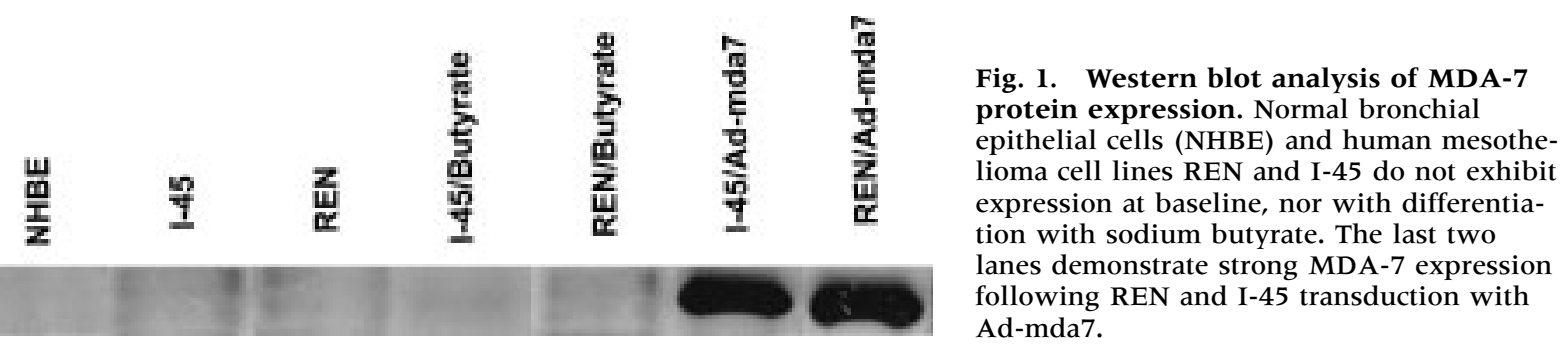

\section{Ad-mda7 Transduction Leads to Over-expression of the MDA-7 Protein in Human Mesothelioma Cells}

REN and I-45 cells were exposed to Ad-mda7 or control Ad-lacZ vectors at an MOI of $3000 \mathrm{vp} / \mathrm{cell}$. Previous studies demonstrated that this dose level resulted in $>90 \%$ transduced cells. Forty-eight hours after treatment, cells were harvested and protein lysates were assayed for MDA-7 protein. Significant expression of MDA-7 protein was noted in both the I-45 and REN cell lines following transfection with the Ad-mda7 vector (Fig. 1).

\section{Over-expression of mda-7 Leads to Death in Human} Mesothelioma Cells

Following transfection with Ad-mda7, cell viability was assayed utilizing a colorimetric (XTT) assay in a dose-response experiment. Figure 2 shows results of REN and I-45 cells treated with $3000 \mathrm{vp} / \mathrm{cell}$ Admda7 or Ad-lacZ for 5 days. Significant cell death $(>50 \%)$ was engendered in both lines following gene transfer $(\mathrm{P}<0.001$, ANOVA).

Mechanism of Death for Mesothelioma Cells Over-expressing MDA-7 is Apoptosis

As an initial evaluation of apoptosis, MPM cells were treated with Ad-mda7 at a dose of $3000 \mathrm{vp} / \mathrm{cell}$. Five days later, Hoescht staining demonstrated

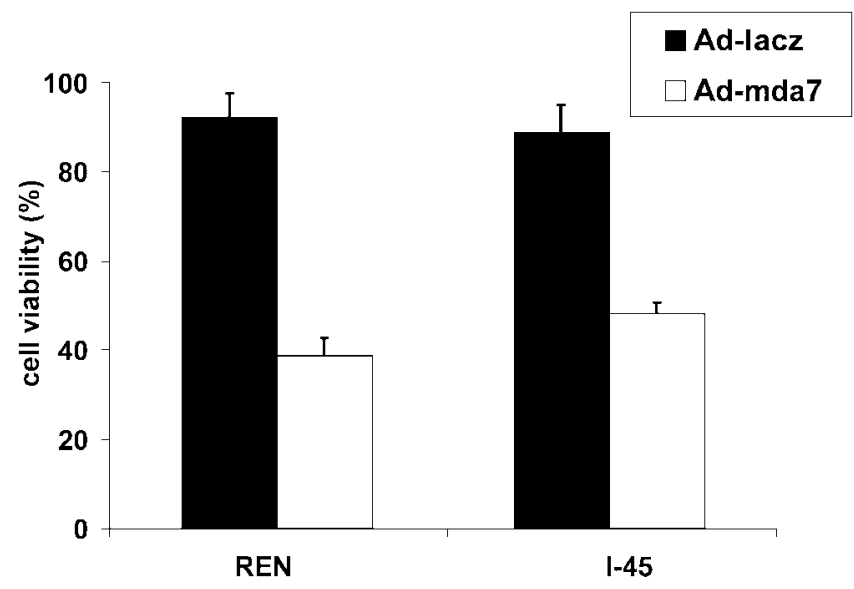

Fig. 2. Cell viability assay for human mesothelioma cell lines REN and I-45 following Ad-mda7 and Ad-lacz exposure ( $3000 \mathrm{vp} / \mathrm{cell}$, 96 hours post exposure). A significant increase in cellular death was noted following Ad-mda7 exposure compared to control $(\mathrm{p}<\mathbf{0 . 0 0 1})$. Results are standardized to untreated cells ( $100 \%$ viability). typical morphologic changes associated with apoptosis, including nuclear condensation and fragmentation (data not shown). To evaluate apoptosis in a more objective fashion REN and I-45 cells were treated with Ad-mda7 $3000 \mathrm{vp} /$ cell, and subGl cell cycle fraction FACS was performed 4 days later. The subGl or apoptotic fraction in REN and I-45 was markedly increased over trivial baseline subGl status by Ad-mda7 transfection (REN 24\%, I-45 $32 \%$ subGl following Ad-mda7 $(p<0.01$, Student's t-test) (Fig. 3).

\section{Ad-mda7 Transduction Leads to Up-regulation of Pro-apoptotic Bax Gene Expression and Caspase Activation in Mesothelioma Cells}

An evaluation of expression of bcl-2 family proteins was performed in an effort to identify any alteration which might have either allowed the cell to progress to apoptosis (anti-apoptotic protein inhibition) or directly engendered apoptosis (over-expression of proapoptotic protein). Expression of p53, as well as of the pro-apoptotic proteins $\mathrm{BAX}$ and $\mathrm{BAK}$, and the antiapoptotic proteins BCL-2 and BCL-XL, was evaluated by Western Blot following treatment of MPM cells with Ad-mda7 or control Ad-lacZ at $3000 \mathrm{vp} / \mathrm{cell}$ for 72 hours. As is shown in Figure $4 \mathrm{~A}$, although no changes are noted in BCL-2, BCL-XL or control actin,

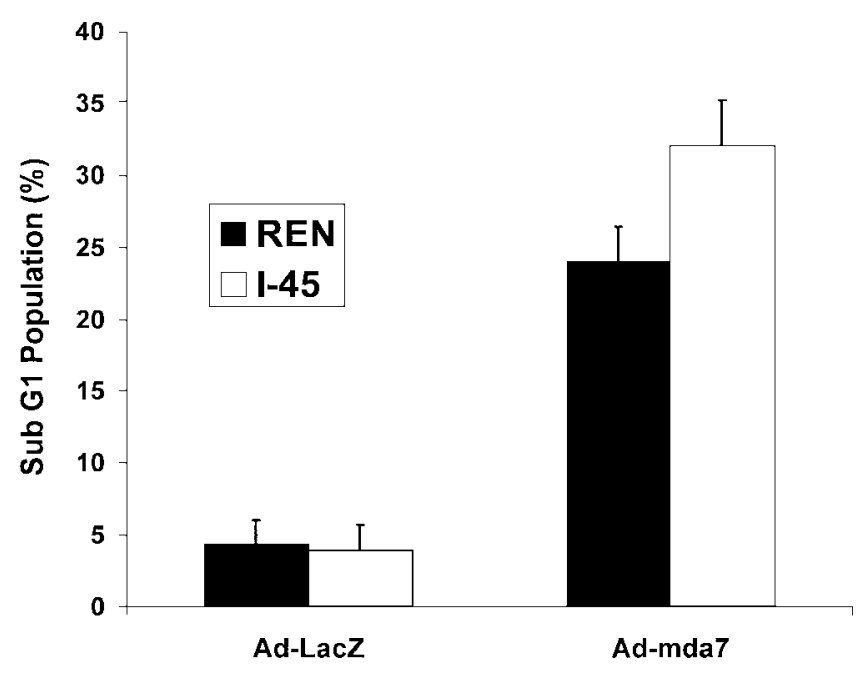

Fig. 3. Apoptosis as measured by subGl FACS analysis in the human mesothelioma cell lines REN and I-45 following Ad-mda7 and Ad-lacZ treatment (3000 vp/cell, 96 hours post exposure). A significant increase in apoptosis was noted following Ad-mda7 exposure compared to control $(p<0.01)$. 

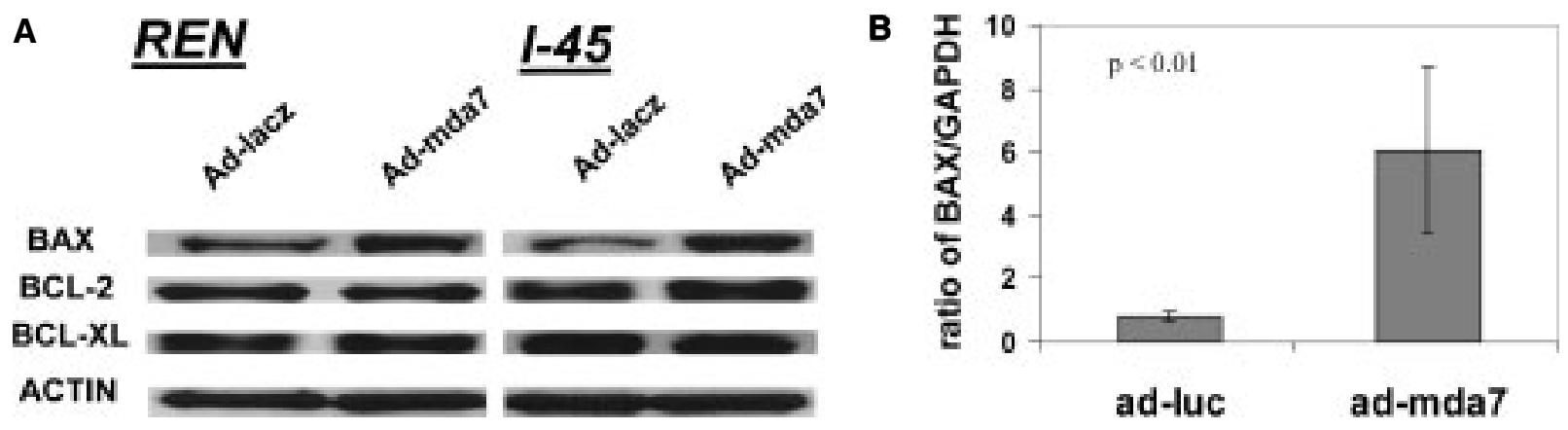

Fig. 4. Western blot analysis of bcl-2 family protein expression following Ad-mda7 exposure. (4A) An increase in BAX expression is noted in both human mesothelioma cell lines following transduction ( 3000 vp/cell, 96 hours post exposure). (4B) A more than 6-fold increase in bax gene expression at the mRNA level following Ad-mda7 exposure is noted by real time PCR in the I-45 mesothelioma cell line.

the expression of the pro-apoptotic protein $\mathrm{BAX}$ is substantially increased over baseline (by densitometry analysis REN $-38 \%$ and I- $45-60 \%$ ). In the I-45 MPM cell line expressing wild-type p53, an increase in p53 is noted, but this is not seen in the REN line, which harbors a mutant p53 gene (Fig. 5). Finally, a significant increase in caspase 3 cleavage is seen in both MPM cell lines exposed to Ad-mda7 when compared to controls (Fig. 6). In figure 4B. bax mRNA level is significantly increased in Ad-mda7 treated cells compared to AdLuc treated controls when compared to GAPDH reference by real-time PCR.

\section{Over-Expression of BCL-XL Protein Abrogates the} Pro-Apoptotic Effect of MDA-7 Over-Expression

Utilizing plasmid transfer techniques, several stable I-45 MPM bcl-xl over-expressing cell lines were developed (22). The degree of apoptotic cellular death following equivalent Ad-mda7 exposure was then compared between a high BCL-XL over-expressing cell line $(\mathrm{I}-45 \mathrm{xl})$ and a control cell line stably transfected with a control plasmid over-expressing green
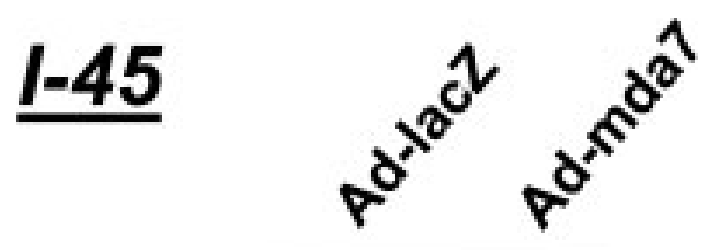

P53

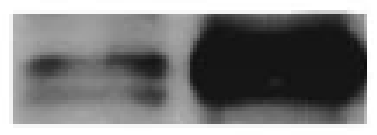

\section{ACTIN}

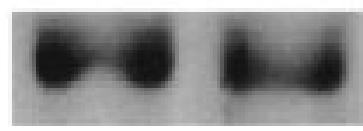

Fig. 5. Western blot demonstrating increase in P53 expression in the p53 wild-type human mesothelioma cell line, I-45, following exposure to Ad-mda7. Exposure to Ad-lacZ is utilized as a control (3000 vp/cell, 96 hours post-exposure). fluorescent protein (I-45gfp). In addition, the ability of Ad-mda7 to lead to eventual caspase 3 cleavage was also evaluated in both control and BCL-XL over-expressing cells. Figure 7 demonstrates the ability of BCL-XL over-expression to inhibit apoptosis in I-45 cell lines following exposure to Ad-mda7, with a significant decrease in sub-Gl fraction from 38.5 to $7.43 \%(p<0.01$, students $t$-test $)$. In addition, both caspase 3 cleavage and caspase 3 activity following Ad-mda7 transfection are diminished by over-expression of bcl-xl as demonstrated in figure 8. Caspase 3 activity was evaluated in the I-45 clones after treatment with Ad-mda7. I-45xl had significantly reduced caspase 3 activity compared to I-45gfp. After addition of DEVD, a caspase inhibitor, both clones exhibit reduced caspase 3 activity, although the inhibition is greater in I-45gfp than in I-45xl.

\section{Discussion}

MPM continues to be a difficult clinical problem. The relative clinical resistance to chemotherapy and

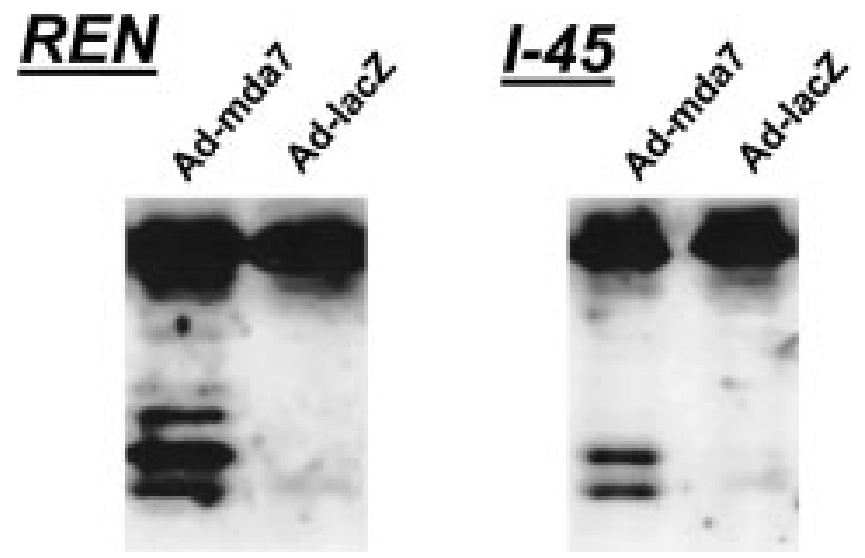

Fig. 6. Western blot analysis of caspase-3 cleavage following Ad-mda7 transduction of human mesothelioma cell lines REN and I-45. Significant cleavage bands are noted following Ad-mda7 transduction with no significant effect noted following control Ad-lacZ exposure. 


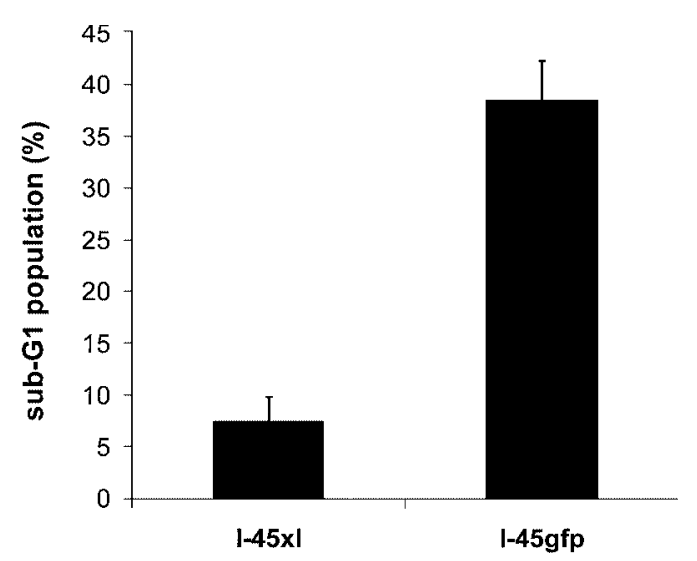

Fig. 7. Comparison of subG 1 FACS analysis of BCL-XL over-expressing clone (I-45xl) and GFP over-expressing control (I-45gfp) human mesothelioma cell line following Ad-mda7 exposure. A significant attenuation of apoptosis is noted in I-45xl.

other conventional treatments would suggest that this tumor is an ideal target for molecular-based therapies. Absence of expression of the pl6 gene seems to be relatively common; but several of the more common molecular targets, such as p53 and ras, are not mutated in MPM cells and tumors, and cytogenetic studies have not yet been instructive (9-11). The forced induction of programmed cell death, or apoptosis, has emerged as a possible strategy for treatment of various tumor types (23). Some of the approaches that have been studied involve the targeting of lesions or pathways specific to tumor cells that may lead to apoptotic cellular death. Unfortunately, little is known about such pathways in this uncommon but deadly neoplasm. It has been proposed that mesothelioma might be relatively resistant to apoptosis once established, and this may in part explain the tumor's insensitivity to conventional therapies. Narasimhan, et al. demonstrated resistance of mesothelioma cells to fairly rigorous
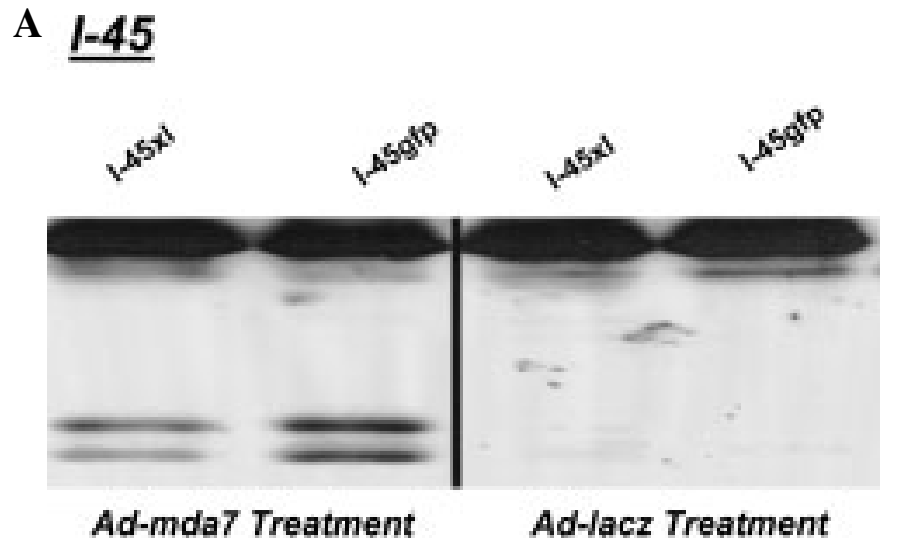

challenge with both oxidant and non-oxidant stimulators of in vitro apoptosis, such as hydrogen peroxide and calcium ionophore (13). Various unconventional in vitro treatments with substances such as crocidilite asbestos, lovastatin, talc and amphotericin have been noted to induce apoptosis in mesothelioma cells, but no significant effort to explain these results at a mechanistic level has been attempted (24-27).

In melanoma, a tumor-suppressor type function has been suggested for $m d a-7$, as progenitor cells express the MDA-7 protein, which is lost with tumoral de-differentiation, and later ectopic expression in HO-1 melanoma tumor cells results in suppression of growth (14). A role for mda-7 as a "classical" tumor suppressor gene, however, seems unlikely. We did not demonstrate expression of MDA-7 protein in our mesothelioma cell lines with differentiation following exposure to sodium butyrate, and $\mathrm{Su}$, et al. did not note baseline mRNA expression in normal breast epithelium in their experiments demonstrating breast carcinoma growth inhibition with MDA-7 over-expression (28). In addition, we have also attempted to induce MDA-7 expression in non-small cell lung carcinoma cell lines (which are also differentiated with exposure to butyrate), and have sought out MDA-7 expression in a number of non-small cell, mesothelioma and normal lung and pleural tissue specimens via immunohistochemistry. Neither in cell line or tissue evaluations were we able to demonstrate MDA-7 expression at the resolution of this technique (data not shown).

The in vitro growth of a number of human and animal tumor cell lines has been inhibited by overexpression of mda-7, including breast, prostate, cervical, colorectal, nasopharyngeal and neurologic neoplasms $(17-20,28)$. Interestingly, the effect on all normal cells reported thus far appears to be absent or greatly attenuated. The growth inhibition due to
B

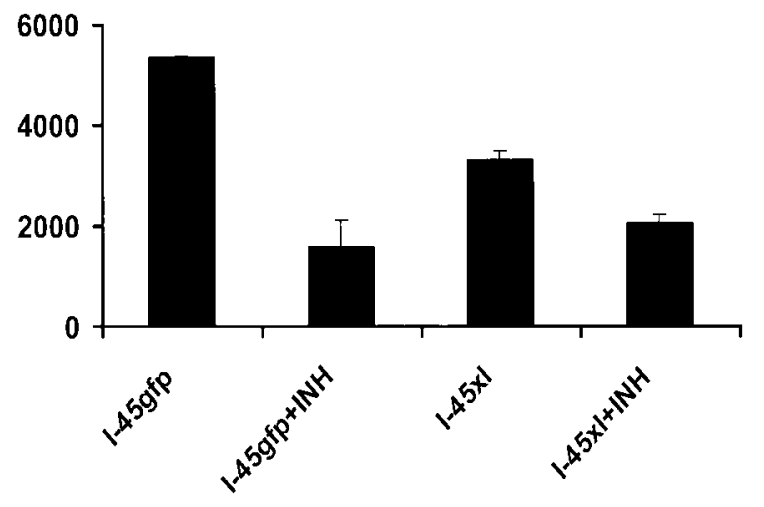

Fig. 8. Caspase-3 activation (cleavage) following Ad-mda7 exposure. (8A) I-45xl and I-45gfp are compared as to functional caspase activity ( $\mathrm{Y}$ axis). Caspase-3 cleavage is attenuated in $\mathrm{I}-45 \mathrm{xl}$ after Ad-mda7 treatment. (8B) Active caspase-3 activity assay results in the I-45 mesothelioma cell line demonstrating abrogation of caspase activation with both inhibitor (INH) following Ad-mda7 exposure in both I-45gfp and I-45xl, as well as an attenuation of caspase-3 activity in I-45xl compared to I-45gfp. 
forced expression of MDA-7 protein has been shown to be related to true apoptosis in breast, and nonsmall cell lung and colorectal carcinoma lines via what appears to be a p53-independent mechanism $(17,19,21)$. In the paper evaluating the effect of MDA-7 over-expression in non-small cell lung carcinoma, an increase in BAX expression, with resultant increases in caspase 3 and 9 activation were noted (19). Although one pathway of bax gene expression is known to be positively regulated by p53 expression, neither $\mathrm{Su}$, et al. (28) nor we noted any difference in the up-regulation of BAX or the rate of cellular growth inhibition and apoptosis between p53 expressing and non-expressing cell lines (mutant or absent). However, in the manuscript by Saeki, et al. p53 expression appeared to be important for the upregulation of BAX (19). These results strongly argue for a p53 independent, bax dependent pathway for release of cytochrome $\mathrm{c}$ from mitochondria and caspase activation in some tumor cells, including MPM. Data demonstrating strong up-regulation of bax expression at both the protein (translational) and mRNA (transcriptional) level in human mesothelioma cells are provided in this study. The mechanism of this bax up-regulation is unknown, but the fact that mda-7 does not share structural or sequence relationships with known transcription factors argues for intermediary cofactors. It is important to note, however, that a BAX-independent caspase-activation pathway may exist in other tumor cell types, as growth suppression and apoptosis of the bax-deficient tumor cell line DU145 has been noted with MDA-7 over-expression (18). In addition, although caspase 3 activation is clearly noted in these mesothelioma cell lines, it is also apparent from studies evaluating effects on the caspase- 3 deficient MCF-7 breast carcinoma cell that this particular caspase is not necessary, as caspase- 9 and others may be equivalent via the ability of BAX to directly affect mitochondrial membrane permeability. In addition, apoptotic stimuli other than BAX up-regulation can certainly trigger caspase activation (28).

In further support of an important role of the bcl-2 family in mda-7 induced apoptosis Su, et al. demonstrated the ability of anti-apoptotic bcl-2 over-expression to selectively inhibit apoptosis in breast carcinoma cells treated with an adenoviral mda-7 gene transfer vector (28). This group theorizes that the increased expression of $\mathrm{BAX}$ is counteracted by the BCL-2, and that the ratio of pro-apoptotic to anti-apoptotic proteins is re-established in this fashion. In contrast to breast carcinoma cell lines, mesothelioma cell lines and tumors very rarely, if ever, express BCL-2, but routinely express BCL-XL and a normal contingent of pro-apoptotic bcl-2 family genes $(12,13)$. We have previously demonstrated the important apoptotic homeostasis maintenance role of BCL-XL expression in mesothelioma cells via experiments in which BCL-XL expression is down-regulated pharmacologically by a histone deacetylase inhibitor. We have demonstrated that decreased expression of BCL-XL alone can lead to apoptosis in mesothelioma cell lines, and that the combination of pharmacologic down-regulation of BCL-XL plus forced adenoviral gene therapy vector over-expression of BAX or BAK leads to additive, and in some cases, synergistic effects on apoptotic cellular death $(22,29)$. When we overexpressed BCL-XL in one of our mesothelioma cell lines via stable plasmid clonal techniques, the effect of Ad-mda7 transduction and MDA-7 over-expression was greatly attenuated. It is pertinent to note that although bax expression was not altered by this change in bcl-xl expression level following transduction, caspase-3 activity as well as cleavage was markedly inhibited. BCL-XL may certainly bind to BAX, but this has been shown to be unnecessary for anti-apoptotic effect. The BCL-XL protein has been shown to bind directly to the porin channel protein VDAC, and prevent cytochrome c release from the mitochondrion, even after pro-apoptotic stimuli, but unlike BCL-2, it does not seem to require dimerization with pro-apoptotic proteins such as BAX to provide for this effect (30-32).

On the basis of these and other groups' results, the direct cancer growth suppressing and apoptosis inducing properties of MDA-7 suggest MDA-7 may prove amenable for developing new and improved cancer therapeutics. Because of the apparent importance in mesothelioma cell lines of BAX expression in MDA-7 induced apoptosis, combinations of therapies such as Ad-mda7 with any others which can decrease BCL-XL expression may provide for additive or synergistic effect.

\section{References}

1. Ong ST, Vogelzang NJ. (1996) Chemotherapy in malignant pleural mesothelioma. A review. J. Clin. Oncol. 14(3): 10071017.

2. Sahmoud T, Postmus PE, van Pottelsberghe C, et al. (1997) Etoposide in malignant pleural mesothelioma: two phase II trails of the EORTC Lung Cancer cooperative Group. Eur. J. Cancer. 33(13): 2211-2215.

3. Antman KH. (1989) Natural history and staging of malignant mesothelioma. Chest. 96(1 Suppl): 93S-95S.

4. Frizelle SP, Rubins JB, Zhou JX, et al. (2000) Gene therapy of established mesothelioma xenografts with recombinant pl6INK4a adenovirus. Cancer Gene Ther. 7(11): 1421-1425.

5. Smythe WR, Hwang HC, Amin KM, et al. (1994) Use of recombinant adenovirus to transfer the herpes simplex virus thymidine kinase (HSVtk) gene to thoracic neoplasms: an effective in vitro drug sensitization system. Cancer Res. 54(8): 2055-2059.

6. Smythe WR, Hwang HC, Elshami AA, et al. (1995) Treatment of experimental human mesothelioma using adenovirus transfer of the herpes simplex thymidine kinase gene. Ann. Surg. 222(1): 78-86.

7. Pass HI, Robinson BW, Testa JR, Carbone M. (1999) Emerging translational therapies for mesothelioma. Chest. 116(6 Suppl): 455S-460S.

8. Sterman DH, Treat J, Litzky LA, et al. (1998) Adenovirusmediated herpes simplex virus thymidine kinase/ganciclovir gene therapy in patients with localized malignancy: results of a phase I clinical trial in malignant mesothelioma. Hum. Gene Ther. 9(7): 1083-1092. 
9. Taguchi T, Jhanwar SC, Siegfried JM, et al. (1993) Recurrent deletions of specific chromosomal sites in $1 p, 3 p, 6 q$, and $9 p$ in human malignant mesothelioma. Cancer Res. 53(18): 4349-4355.

10. Mor O, Yaron P, Huszar M, et al. (1997) Absence of p53 mutations in malignant mesotheliomas. Am. J. Respir. Cell Mol. Biol. 16(1): 9-13.

11. Nishiyama Y, Suwa H, Okamoto K, (1995) Low incidence of point mutations in $\mathrm{H}-, \mathrm{K}$ - and $\mathrm{N}$-ras oncogenes and p53 tumor suppressor gene in renal cell carcinoma and peritoneal mesothelioma of Wistar rats induced by ferric nitrilotriacetate. Jpn. J. Cancer Res. 86(12): 1150-1158.

12. Soini Y, Kinnula V, Kaarteenaho-Wiik R, et al. (1999) Apoptosis and expression of apoptosis regulating proteins bcl-2, mcl-1, bcl-X, and bax in malignant mesothelioma. Clin. Cancer Res. 5(11): 3508-3515.

13. Narasimhan SR, Yang L, Gerwin BI, Broaddus VC. (1998) Resistance of pleural mesothelioma cell lines to apoptosis: relation to expression of Bcl-2 and Bax. Am. J. Physiol. 275(1 Pt 1): L165-L171.

14. Jiang H, Lin J, Su Z-Z, et al. (1995) Subtraction hybridization identifies a novel melanoma differentiation associated gene, mda-7, modulated during human melanoma differentiation, growth and progression. Oncogene. 11: 2477-2486.

15. Madireddi MT, Dent P, Fisher PB. (2000) Regulation of mda7 gene expression during human melanoma differentiation. Oncogene. 19(10): 1362-1368.

16. Ekmekcioglu S, Ellerhorst J, Mhashilkar AM, et al. (2001) Down-regulated melanoma differentiation associated gene (mda-7) expression in human melanomas. Intl. J. Cancer. 94(1): 54-59.

17. Jiang H, Su Z-Z, Lin JJ, et al. (1996) The Melanoma Differentiation Associated Gene MDA-7 Suppresses Cancer Cell Growth. Proc. Natl. Acad. Sci. USA. 93: 9160-9165.

18. Su ZZ, Madireddi MT, Lin JJ, et al. (1998) The cancer growth suppressor gene mda-7 selectively induces apoptosis in human breast cancer cells and inhibits tumor growth in nude mice. Proc. Natl. Acad. Sci. USA. 95(24): 14400-14405.

19. Saeki T, Mhashilkar A, Chada S, et al. (2000) Tumor-suppressive effects by adenovirus-mediated mda-7 gene transfer in non-small cell lung cancer in vitro. Gene Ther. 7: 2051-2057.

20. Madireddi MT, Su ZZ, Young CS, et al. (2000) Mda-7, a novel melanoma differentiation associated gene with promise for cancer gene therapy. Adv. Exp. Med. Biol. 465: 239-261.
21. Mhashilkar AM, Schrock RD, Hindi M, et al. (2001) Melanoma differentiation associated gene (mda-7): a novel anti-tumor gene for cancer gene therapy. Mol. Med. 7(4): 271-282.

22. Cao XX, Mohiuddin I, Ece F, et al. (2001) Histone deacetylase inhibitor down-regulation of bcl-xl gene expression leads to apoptotic cell death in mesothelioma. Am. J. Respir. Cell Mol. Biol. 25(5): 562-568.

23. Korsmeyer SJ. (1999) BCL-2 gene family and the regulation of programmed cell death. Cancer Res. 59(7 Suppl): 1693s1700 s.

24. Nasreen N, Mohammed KA, Dowling PA, et al. (2000) Talc induces apoptosis in human malignant mesothelioma cells in vitro. Am. J. Respir. Crit. Care Med. $161(2$ Pt 1): 595-600.

25. Rubins JB, Greatens T, Kratzke RA, et al. (1998) Lovastatin induces apoptosis in malignant mesothelioma cells. Am. J. Respir. Crit. Care Med. 157(5 Pt 1): 1616-1622.

26. Broaddus VC, Yang L, Scavo LM, et al. (1996) Asbestos induces apoptosis of human and rabbit pleural mesothelial cells via reactive oxygen species. J. Clin. Invest. 98(9): 2050-2059.

27. Marklund L, Henriksson R, Grankvist K. (2000) Amphotericin $\mathrm{B}$-induced apoptosis and cytotoxicity is prevented by the $\mathrm{Na}+, \mathrm{K}+, 2 \mathrm{Cl}(-)$-cotransport blocker bumetanide. Life Sci. 66(23): L319-L324.

28. Su Z, Madireddi M, Lin J, et al. (1998) The cancer growth suppressor gene mda-7 selectively induces apoptosis in human breast cancer cells and inhibits tumor growth in nude mice. Proc. Natl. Acad. Sci. USA. 95: 14400-14405.

29. Mohiuddin I, Cao X, Fang B, et al. (2001) Significant augmentation of pro-apoptotic gene therapy by pharmacologic bcl-xl down-regulation in mesothelioma. Cancer Gene Ther. 8: 547-554.

30. Shimizu S, Shinohara Y, Tsujimoto Y. (2000) Bax and Bcl-xL independently regulate apoptotic changes of yeast mitochondria that require VDAC but not adenine nucleotide translocator. Oncogene. 19(38): 4309-4318.

31. Kim CN, Wang X, Huang Y, et al. (1997) Overexpression of Bcl-X(L) inhibits Ara-C-induced mitochondrial loss of cytochrome $\mathrm{c}$ and other perturbations that activate the molecular cascade of apoptosis. Cancer Res. 57(15): 3115-3120.

32. Gottlieb E, Vander Heiden MG, Thompson CB. (2000) Bcl$x(L)$ prevents the initial decrease in mitochondrial membrane potential and subsequent reactive oxygen species production during tumor necrosis factor alpha-induced apoptosis. Mol. Cell Biol. 20(15): 5680-5689. 